\title{
Molecular mechanisms of blister formation in bullous impetigo and staphylococcal scalded skin syndrome
}

\author{
Yasushi Hanakawa, ${ }^{1}$ Norman M. Schechter, ${ }^{1}$ Chenyan Lin, ${ }^{1}$ Luis Garza, ${ }^{1}$ Hong Li, ${ }^{1}$ \\ Takayuki Yamaguchi, ${ }^{2}$ Yasuyuki Fudaba, ${ }^{2}$ Koji Nishifuji, ${ }^{3}$ Motoyuki Sugai, ${ }^{2}$ \\ Masayuki Amagai, ${ }^{3}$ and John R. Stanley ${ }^{1}$
}

${ }^{1}$ Department of Dermatology, University of Pennsylvania School of Medicine, Philadelphia, Pennsylvania, USA
2Department of Bacteriology, Hiroshima University Graduate School of Biomedical Sciences, Hiroshima, Japan
${ }^{3}$ Department of Dermatology, Keio University School of Medicine, Tokyo, Japan

Bullous impetigo due to Staphylococcus aureus is one of the most common bacterial infections of man, and its generalized form, staphylococcal scalded skin syndrome (SSSS), is a frequent manifestation of staphylococcal epidemics in neonatal nurseries. Both diseases are mediated by exfoliative toxins (ETs), which show exquisite pathologic specificity in blistering only the superficial epidermis. We show that these toxins act as serine proteases with extremely focused molecular specificity to cleave mouse and human desmoglein 1 (Dsg1) once after glutamic acid residue 381 between extracellular domains 3 and 4 . Mutation of the predicted catalytically active serine to alanine completely inhibits cleavage. The mutated ETs bind specifically to Dsg1 by immunofluorescence colocalization and by coimmunoprecipitation. Thus, ETs, through specific recognition and proteolytic cleavage of one structurally critical peptide bond in an adhesion molecule, cause its dysfunction and allow S. aureus to spread under the stratum corneum, the main barrier of the skin, explaining how, although they circulate through the entire body in SSSS, they cause pathology only in the superficial epidermis.

J. Clin. Invest. 110:53-60 (2002). doi:10.1172/JCI200215766.

\section{Introduction}

Understanding the pathology resulting from Staphylococcus aureus infection is of great interest in medicine because of the organism's common and increasing prevalence in humans, its growing bacterial resistance, and its ability to cause serious and life-threatening disease (1). Toxins contribute in a major way to the pathogenicity of $S$. aureus, as they do with many other bacteria (2).

$S$. aureus frequently infects the skin. In fact, the most common bacterial infection of children is impetigo, which accounts for approximately $10 \%$ of all skin problems in children (3). Of these impetigo patients, about $30 \%$ have bullous impetigo, which is caused by S. aureus strains that produce exfoliative toxins (ETs). In patients discharged from the newborn nursery, over $30 \%$ may be colonized with S. aureus, and organisms that produce ETs are the major cause of neonatal nursery outbreaks of staphylococcal disease $(1,4)$. Newborns, young children, and adults with renal failure and/or who are

Received for publication April 22, 2002, and accepted in revised form May 29, 2002.

Address correspondence to: John R. Stanley, Department of Dermatology, University of Pennsylvania, School of Medicine, 211 Clinical Research Building, 415 Curie Boulevard, Philadelphia, Pennsylvania 19104, USA. Phone: (215) 898-3240; Fax: (215) 573-2033; E-mail: jrstan@mail.med.upenn.edu.

Conflict of interest: No conflict of interest has been declared. Nonstandard abbreviations used: exfoliative toxin (ET); staphylococcal scalded skin syndrome (SSSS); desmoglein 1 (Dsg1); Tris-buffered saline (TBS); horseradish peroxidase (HRP); pemphigus foliaceus (PF); extracellular cadherin domain (EC). immunocompromised may develop a generalized form of bullous impetigo called staphylococcal scalded skin syndrome (SSSS). In this disease, bullae and erosions develop over a large area of the skin surface, resulting in the death of probably less than $3 \%$ of affected children but up to $60 \%$ of affected adults $(5,6)$.

The now-classic experiments of Melish et al. in the early 1970's established that ET produced by S. aureus causes the blistering in bullous impetigo and SSSS (7-9). Passive transfer of the toxin to neonatal mice caused the same skin lesions seen in humans, namely a blister in the superficial living epidermis caused by separation of the keratinocytes in the granular cell layer due to splitting of the desmosomes as either a primary or secondary event $(10,11)$. Presumably, this type of blister allows the bacteria to spread under and circumvent the stratum corneum, a major barrier that prevents infection of the skin. Two major ETs, ETA and ETB, which share 40\% identity in amino acid sequence, have been identified and cloned $(12,13)$. Recently, a third ET (termed ETD) that causes identical epidermal blisters has been identified (T. Yamaguchi et al., unpublished observations). In bullous impetigo, blisters occur at the site of infection with S. aureus, and are caused by local production of ET, whereas in SSSS the toxin is produced at the site of infection (not necessarily in the skin) and causes generalized blistering due to its systemic circulation. The latter disease underscores the exquisite specificity of ETs that, even when they circulate through the entire body, cause pathology only in the superficial epidermis.

The pathophysiologic basis of this specificity has been a mystery for over 30 years, even though recent 
crystal structure and amino acid sequences of ETA and ETB have suggested that they are "atypical" glutamatespecific serine proteases. The term "atypical" was used because some crystal structures of ETA and ETB have shown that the presumed catalytic site is not configured properly to be active, perhaps requiring activation by binding to a specific substrate or receptor (14-17). On the other hand, it has been argued that the toxicity of ETs may not be as serine proteases at all (e.g., data has suggested that these ETs are poorly inhibited by serine protease inhibitors), but as superantigens or through less-direct mechanisms such as release of proteases by the epidermis (reviewed in refs. 1, 18). Furthermore, until very recently, proteolysis of a relevant biological target had not been demonstrated.

Very recently, we identified a relevant substrate of ETA, ETB, and ETD to be desmoglein 1 (Dsg1), a transmembrane glycoprotein of desmosomes in the cadherin gene superfamily $(19,20)$ (T. Yamaguchi et al., unpublished observations). Dsg1, but not the closely related Dsg3 or E-cadherin, is cleaved by ETs. Furthermore, Dsg1 is a relevant substrate because antibodies in the autoimmune disease pemphigus foliaceus (PF) target Dsg1 and cause clinical and histologic blisters in mouse and man identical to those seen in bullous impetigo and SSSS. These data imply that targeting of Dsg1 can result in the pathology characteristic of bullous impetigo and SSSS.

Here we demonstrate that ETA, ETB, and ETD act as glutamic acid-specific serine proteases with unusually focused specificity that results in a single cleavage of Dsg1, leading to its functional inactivation. The pathophysiology of bullous impetigo and SSSS can thus be attributed to hydrolysis of a single peptide bond, presumably in a functionally critical area in Dsg1.

\section{Methods}

Recombinant ETs. The expressed proteins, vectors, and their hosts are summarized in Table 1. Shuttle vector pCE104, containing ETA and ETA $\mathrm{C}_{\mathrm{mu}}$, was from Patrick Schlievert (Department of Microbiology, University of Minnesota Medical School, Minneapolis, Minnesota, USA) (14). ETA $A_{\text {mu }}$ was constructed from these vectors with the QuikChange Site-Directed Mutagenesis Kit (Stratagene, La Jolla, California, USA). ETB A was cloned by PCR using PCE104-ETB (20) as a template. PCR was also used to add nucleotides encoding peptide tags, V5His (Invitrogen Corp., Carlsbad, California, USA) or 3X FLAG (Sigma-Aldrich, St. Louis, Missouri, USA), to the $3^{\prime}$ end of these ET constructs. ETAV5His, ETA $\mathrm{C}_{\mathrm{mu}}-$ V5His, ETA $\mathrm{A}_{\mathrm{mu}}-\mathrm{V} 5$ His, ETB-V5His, ETB-3X FLAG, and ETB $\mathrm{A}_{\mathrm{mu}}-\mathrm{V} 5$ His were subcloned into the HindIII and EcoRI sites of pCE104. DNA was transformed into the $S$. aureus strain RN4220 (provided by Patrick Schlievert) via electropolation. The supernatant from RN4220 transformed with these plasmids was precipitated with $85 \%$ ammonium sulfate. Precipitated ETs were dialyzed against PBS with $1 \mathrm{mM} \mathrm{CaCl}_{2}$ (PBS-Ca). His-tagged ETs were purified on Ni-NTA
Table 1

Vectors and hosts used for expression of ETs and desmogleins

\begin{tabular}{|c|c|c|}
\hline Protein & Vector & Host \\
\hline ETA & pCE104-ETA-V5His & S. aureus \\
\hline ETA C $_{m u}$ & pCE104-ETA $C_{m u}-V 5 H$ is & S. aureus \\
\hline ETA $A_{m u}$ & pCE104-ETA A $A_{m u}-V 5$ His & S. aureus \\
\hline ETB & pCE104-ETB-V5His & S. aureus \\
\hline ETB-FLAG & pCE104-ETB-3X FLAG & S. aureus \\
\hline ETB $A_{m u}$ & pCE104-ETB $A_{m u}-V 5 H i s$ & S. aureus \\
\hline ETD & PQE70-ETD-His & E. coli \\
\hline ETD A $A_{m u}$ & PQE70-ETD A $A_{m u}-H$ is & E. coli \\
\hline hDsg1E & Baculo-hDsg1E & Insect cell (High Five) \\
\hline mDsg1E & Baculo-mDsg1E & Insect cell (High Five) \\
\hline Dsg1FLAG & Ax-mDsg1F & Keratinocytes $(\mathrm{HaCaT})$ \\
\hline Dsg3FLAG & Ax-mDsg3F & Keratinocytes ( $\mathrm{HaCaT})$ \\
\hline
\end{tabular}

Baculo, baculovirus vector; $A x$, adenovirus vector.

columns (QIAGEN Inc., Valencia, California, USA) using the manufacturer's procedure and then dialyzed against PBS-Ca. ET concentrations were estimated with a protein assay kit from Bio-Rad Laboratories Inc. (Hercules, California, USA).

ETD was cloned by PCR from a patient sample ( $T$. Yamaguchi et al., unpublished data), and ETD A $\mathrm{mu}_{\mathrm{mu}}$ was cloned by PCR mutagenesis. The amplified DNA fragments were cloned into PQE70 in order to express the fusion protein with a $6 x \mathrm{His}$ tag sequence on the carboxy terminus in E. coli. The recombinant proteins were purified using Ni-NTA resin according to the manufacturer's protocol (QIAGEN Inc.).

For comparison of mutant to wild-type ETs (see Figure 2), the supernatants from transformed RN4220 staphylococci were used, with the amount of ETs normalized by Coomassie blue staining of SDS-PAGE gels in which the ETs were the major band and accounted for over $90 \%$ of the total protein.

Cell culture and transduction with adenovirus vectors. $\mathrm{HaCaT}$ cell keratinocytes cultured in DMEM with 10\% FBS were transduced with recombinant adenovirus vectors Ax-mDsg1F and Ax-mDsg3F (20), encoding mouse Dsg1 and Dsg3, respectively, with carboxy-terminal FLAG tags (Table 1). After 24 hours, the cells were incubated with recombinant ETA, ETB, or ETD in culture media for 10 minutes. Then the cells were washed with PBS-Ca, extracted, and used for immunoprecipitation or immunoblotting.

In vitro digestion of recombinant Dsg1 with ETs. The entire extracellular domain of human or mouse recombinant Dsg1 with an E tag (hDsg1E and mDsg1E) on the carboxyl terminus was produced as a secreted protein by baculovirus, as previously described (21). (These constructs also contained a His tag on the carboxy terminus.) Approximately $1 \mu \mathrm{g}$ of mouse or human Dsg1 purified with a TALON (BD Biosciences Clontech, San Jose, California, USA) or E-tag column (Amersham Biosciences Inc., Piscataway, New Jersey, USA) was incubated with recombinant ETA, ETB, or ETD in Trisbuffered saline with $1 \mathrm{mM} \mathrm{CaCl}$ (TBS-Ca) for 1 hour at 
$37^{\circ} \mathrm{C}$. The digested samples were subjected to SDSPAGE, transferred to PVDF membranes, and stained using Coomassie brilliant blue. Stained bands were cut out and sequenced by Edman degradation at the Protein Microchemistry Facility of The Wistar Institute (Philadelphia, Pennsylvania, USA).

Antibodies. Anti-E-tag mouse monoclonal antibody conjugated with horseradish peroxidase (HRP; Amersham Biosciences Inc.), anti-V5-tag mouse monoclonal antibody (Invitrogen Corp.), anti-His-tag mouse monoclonal antibody (QIAGEN Inc.), anti-FLAG-tag mouse monoclonal antibody (M2; Sigma-Aldrich), anti-ETA sheep polyclonal antibody (Toxin Technology Inc., Sarasota, Florida, USA), anti-ETD rabbit polyclonal antibody (T. Yamaguchi, unpublished observations), and PF serum (anti-Dsg1) were used for immunoblotting and immunohistochemistry. HRP-conjugated anti-IgG antibodies of the appropriate specificity (Bio-Rad Laboratories Inc.) were used for immunoblotting. Anti-human antibody conjugated with Alexa Fluor 495 and antimouse antibody conjugated with Alexa Fluor 590 (Molecular Probes Inc., Eugene, Oregon, USA) were used for immunohistochemistry.

Ex vivo binding of ETA $A_{m u}$ to mouse epidermis, and immunohistochemistry. Mouse tail or paw skin was incubated in DMEM at a ratio of 1:1 with 10\% FBS and ETA $\mathrm{A}_{\mathrm{mu}}-\mathrm{V} 5 \mathrm{His}(\sim 83 \mu \mathrm{M})$ in PBS-Ca at $4^{\circ} \mathrm{C}$ for 12 hours and then cryosectioned. After being fixed with $4 \%$ paraformaldehyde in PBS-Ca, specimens were blocked with 1\% BSA in PBS-Ca. Blocked specimen was incubated with anti-V5 antibody followed by anti-mouse antibody conjugated with Alexa Fluor 590, and then stained with PF serum followed by anti-human antibody conjugated with Alexa Fluor 495. Stained sections were photographed using confocal microscopy (MRC 1024; Bio-Rad Laboratories Inc.). For controls, skin was first incubated with wild-type ETA or an irrelevant toxin (staphylococcal enterotoxin B with a V5 tag), then stained with PF serum or anti-V5 antibody, respectively. These controls showed no staining (not shown).

Immunoprecipitation. After incubation with ETs, adenovirus-infected cells were extracted with $1 \%$ Triton X-100 in TBS-Ca with protease inhibitors $(2 \mu \mathrm{g} / \mathrm{ml}$ aprotinin, $2 \mu \mathrm{g} / \mathrm{ml}$ leupeptin, and $1 \mathrm{mM}$ PMSF) for immunoprecipitation. Supernatants of High Five insect cells (Invitrogen Corp.) transduced with baculovirus encoding $\mathrm{hDsg} 1 \mathrm{E}$ were incubated with ETs, then used directly for immunoprecipitation. FLAG-tagged proteins were precipitated with anti-FLAG agarose (Sigma-Aldrich), and E-tagged proteins were precipitated with anti-E-tag Sepharose (Amersham Biosciences Inc.) at $4^{\circ} \mathrm{C}$ for 1 hour. Immunoprecipitates were washed ten times with $1 \%$ Triton X-100 in TBS-Ca, then eluted with Laemmli sample buffer at $100^{\circ} \mathrm{C}$.

Immunoblotting. Cell extracts or immunoprecipitates in Laemmli sample buffer were separated by $4-20 \%$ or $10 \%$ Tris-glycine SDA-PAGE (Bio-Rad Laboratories Inc. or Invitrogen Corp.), then transferred to nitrocellulose a

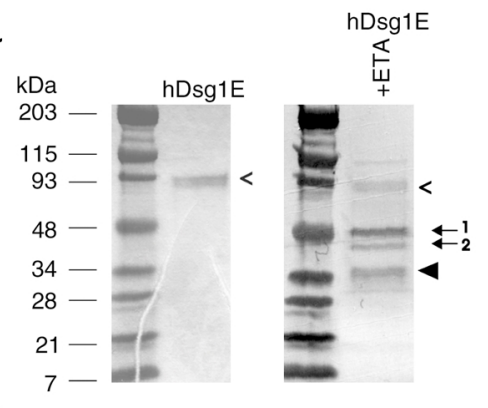

d

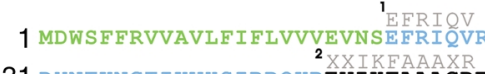

31 DYNTKNGTIKWHSIRRQKREWIKFAAACRE

61 GEDNSKRNPI . . .
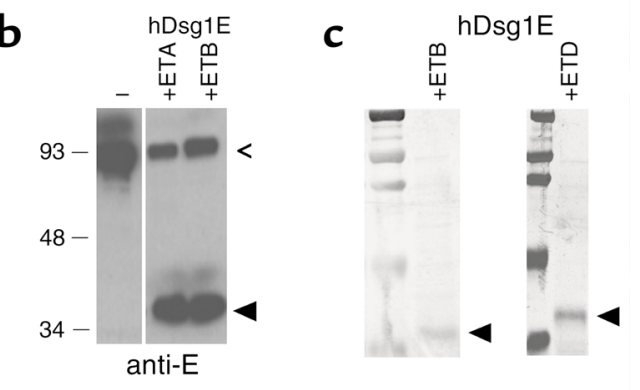

e

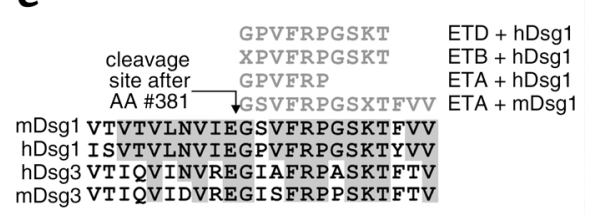

\section{Figure 1}

Cleavage site of human and mouse Dsg1 by ETs. (a) Coomassie brilliant blue staining of SDS-PAGE of purified hDsg1E (left panel) and its ETA cleavage products (right panel). Open arrowhead shows a closely spaced doublet at the predicted molecular weight of $84 \mathrm{kDa}$, representing the proprotein and mature protein. Cleavage results in products with approximate molecular weights of $50 \mathrm{kDa}$ (arrow 1 ), $45 \mathrm{kDa}$ (arrow 2), and $34 \mathrm{kDa}$ (filled arrowhead). (b) Immunoblotting with anti-E-tag antibodies of hDsg1E cleaved by ETA and ETB identifies the $34-\mathrm{kDa}$ band (filled arrowhead) as the carboxy-terminal fragment. Open arrowhead indicates uncleaved hDsg1E. (c) Coomassie blue staining of SDS-PAGE of the carboxy-cleavage fragments of hDsg1E (filled arrowheads) cleaved by ETB or ETD and purified on an anti-E-tag Sepharose column. (d) Peptide sequencing (residues following "1" in light gray) of the 50-kDa cleavage product (arrow 1 in a) showed the amino acid sequence of the amino terminus of hDsg1E lacking the signal peptide (residues in green) but still containing the propeptide (residues in blue). Peptide sequencing (residues following "2" in light gray) of the 45-kDa peptide (arrow 2 in a) identifies the amino terminus of mature hDsg1 (residues in black). (e) Cleavage site in Dsg1. Amino-terminal peptide sequencing (residues in gray) of the 34-kDa carboxy terminus of hDsg1E (filled arrowheads in $\mathbf{a}$ and $\mathbf{c}$ ) and mDsg1E (SDS-PAGE not shown) cleaved by ETs. Peptide sequence in black indicates mouse and human Dsg1 and Dsg3 around the cleavage site. Shading shows peptide identity. AA, amino acid. 


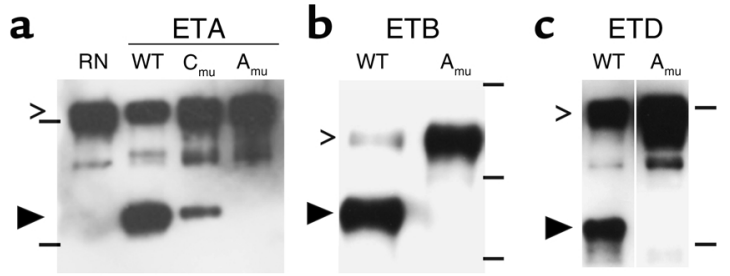

Figure 2

Point mutation of serine 195 (chymotrypsin numbering), the presumed catalytically active serine, of ETs inhibits cleavage of Dsg1. (a) Anti-E-tag antibody immunoblot of SDS-PAGE of hDsg1E incubated with RN4220 staphylococcal vector supernatant (RN), wildtype (WT) ETA, ETA C mu (serine 195 mutated to cysteine), and ETA $A_{\text {mu }}$ (serine 195 mutated to alanine) shows markedly decreased cleavage with ETA $C_{m u}$ compared with wild-type ETA. ETA $A_{m u}$ shows no catalytic activity. Horizontal lines indicate migration of molecular weight markers of $83 \mathrm{kDa}$ (top) and $32 \mathrm{kDa}$. (b) Anti-FLAG-tag immunoblots of anti-FLAG-tag immunoprecipitates of extracts of mDsg1-FLAG adenovirus-transduced cells that were incubated with ETB or ETB $A_{m u}$. ETB $A_{m u}$ shows no cleavage. Horizontal lines, from top, indicate migration of molecular weight markers of $203 \mathrm{kDa}, 115 \mathrm{kDa}$, and $93 \mathrm{kDa}$. (c) Anti-E-tag antibody immunoblot of SDS-PAGE of hDsg1E incubated with ETD and ETD $A_{m u}$. ETD $A_{m u}$ does not cleave hDsg1. Horizontal lines, from top, indicate migration of molecular weight markers of $83 \mathrm{kDa}$ and 34 kDa. Uncleaved Dsg1 (open arrowhead) and its carboxy-terminal cleavage product (filled arrowhead) are indicated.

sheets (Trans-Blot; Bio-Rad Laboratories Inc.). The sheets were incubated for 1 hour in blocking buffer $(5 \%$ fat-free milk powder in PBS). The first antibody, diluted in blocking buffer, was applied for 1 hour at room temperature. After two washes with $0.1 \%$ Tween 20 in PBS, the sheets were incubated with HRP-conjugated secondary antibody diluted in blocking buffer. In some experiments, only one antibody, HRP-conjugated antiETA, was used. After the blots were washed, the signals were detected with chemiluminescence (ECL or ECL Plus, Amersham Biosciences Inc.).

\section{Results}

ETs cleave human and mouse Dsg1 at a unique site. Previous studies of proteolysis of Dsg1 by ETs identified only a small carboxy-terminal fragment, leaving the extent of degradation uncertain. To determine whether there is one specific cleavage site as opposed to more general proteolytic degradation, the extracellular domain of human Dsg1, containing an $\mathrm{E}$ tag (hDsg1E) on the carboxy terminus, was purified and cleavage was characterized by SDS-PAGE and amino-terminal sequence analysis. hDsg1E was produced by baculovirus in High Five insect cells (21). The recombinant protein was isolated from insect cell supernatant with anti-E-tag Sepharose and elution with excess $\mathrm{E}$ peptide. Coomassie blue staining of the peptide-eluted material resolved by SDS-PAGE (Figure 1a, left) indicates a closely spaced doublet at the predicted molecular weight of $84 \mathrm{kDa}$. The isolated hDsg1E was incubated for 1 hour with ETA at $37^{\circ} \mathrm{C}$, and the products were separated by SDS-
PAGE (Figure 1a, right). Two major bands of approximately $50 \mathrm{kDa}$ (arrow marked 1) and $34 \mathrm{kDa}$ (filled arrowhead) were detected by Coomassie blue staining, suggesting that hDsg1E was cleaved at a single site. Immunoblotting with anti-E-tag antibodies of hDsg1E digests identified the 34-kDa band as the carboxy-terminal fragment (Figure 1b). Amino terminal peptide sequencing of this fragment shown in Figure 1a (right side, arrowhead) was then used to determine the exact cleavage site, which was after glutamic acid residue 381 (as counted from the initiating methionine of hDsg1) (Figure 1e). A similar study showed that ETA cleaved mouse Dsg1 at the same site (Figure 1e).

To determine the site of cleavage by ETB and ETD in hDsg1, we incubated unpurified hDsg1E (obtained directly from baculovirus supernatant) with these ETs, then purified the carboxy terminus with anti-E-tag Sepharose. Coomassie blue staining of the resulting purified carboxy-cleavage fragments separated on SDSPAGE is shown in Figure 1c. Amino-terminal peptide sequencing of these fragments indicated that the ETB and ETD cleavage site in Dsg1 was identical to that produced by ETA (Figure 1e). These data are consistent with structural models that suggest that ETA and ETB might cleave a substrate after a glutamic acid residue.

Amino terminal sequence analysis of the slower migrating 50-kDa band formed by cleavage of hDsg1E (Figure 1a, arrow marked 1) revealed the amino terminus of hDsg1E lacking the signal peptide (which was presumably processed by the insect cells) but still containing the propeptide (Figure 1d). Sequencing of the band shown immediately below the 50-kDa band (Figure 1a, arrow marked 2) indicated that it was the amino terminus of the hDsg1E mature protein (Figure 1d). These data show that the signal peptide of hDsg1E produced by baculovirus in insect cells is properly processed, and confirm that the sequence previously predicted by computer modeling to be the signal peptide in hDsg1 (Figure 1d, shown in green) is correct. The data also show that the proprotein produced by baculovirus in insect cells is not efficiently processed. However, both the precursor and mature proteins are efficiently cleaved by ETA at the same site.

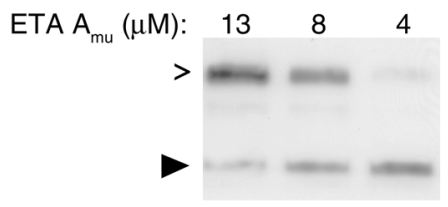

\section{Figure 3}

ETA $A_{m u}$ inhibits cleavage of Dsg1 by ETA. Anti-E-tag immunoblot of hDsg1E (open arrowhead) and its carboxy-terminal cleavage product (filled arrowhead). ETA $A_{m u}$ at a concentration of $13 \mu \mathrm{M}$ incubated with hDsg1E inhibits cleavage by subsequent addition of wild-type ETA, but at $4 \mu \mathrm{M}$ does not. At $8 \mu \mathrm{M}$, about half of the Dsg1 is free to be cleaved. $K_{\mathrm{d}}$ can then be roughly estimated to be $8 \mu \mathrm{M}$. ETA $\mathrm{A}_{\mathrm{mu}}$ in the concentrations shown (all in large excess of that of hDsg1E) was incubated with hDsg 1 at $25^{\circ} \mathrm{C}$ for 60 minutes, then wild-type ETA was added for a 20-minute incubation before SDS-PAGE. 
Taken together, the data in Figure 1 demonstrate that ETA, ETB, and ETD cleave hDsg1 at the same site, and that ETA cleaves $\mathrm{mDsg} 1$ at the homologous site. These ETs are known not to cleave the closely related Dsg3 $(19,20)$. Sequence alignment of human and mouse Dsg1 and Dsg3 (Figure 1e) indicated marked homology (17 of 18 identical residues) of mouse and human Dsg1, but divergence of Dsg3, around the cleavage site.

Serine 195 of ETs is critical for proteolysis of Dsg1. The inference from structural studies of ETA and ETB is that the serine residue at position 195 in ETA and 186 in ETB corresponds to the catalytically active serine 195 of chymotrypsin. Furthermore, like chymotrypsin, ETA and ETB have a functional catalytic triad consisting of serine $195 / 186 / 195$, histidine $72 / 65 / 57$, and aspartic acid 120/114/102 (ETA/ETB/chymotrypsin numbering; subsequent numbering will be for chymotrypsin only). To demonstrate that this serine is critical in the cleavage of hDsg1, it was mutated to a cysteine (resultant mutant ETA $\mathrm{C}_{\mathrm{mu}}$ ). Incubation of ETA $\mathrm{C}_{\mathrm{mu}}$ with Dsg1 at $37^{\circ} \mathrm{C}$ for 1 hour demonstrated a markedly decreased rate of cleavage compared with wild-type ETA (Figure 2). Assuming this activity was likely mediated by the cysteine sulfhydryl group, similar to what has been found in a similar mutant of trypsin (22), we mutated serine 195 to alanine $\left(\right.$ ETA $\left._{\mathrm{mu}}\right)$. The ETA $\mathrm{A}_{\mathrm{mu}}$ did not cleave Dsg1 (Figure 2). We similarly showed that mutation of serine 195 to alanine in ETB and ETD inhibited cleavage of Dsg1 (Figure 2).

The observations that substitution of serine 195 with both cysteine and alanine inhibit cleavage, and that the mutant ETs still specifically bind Dsg1 (see below), demonstrate that serine 195 is necessary for efficient catalytic cleavage of Dsg1.

Binding of ETs to Dsg1. Crystal structural studies of ETA have suggested that it may be an inactive enzyme due to an inappropriate alignment of certain residues forming the active site. It has been postulated that as a consequence, ETA may have to bind to its specific substrate or to a receptor in order to become catalytic $(14-16,23)$. (However, for an opposing view, see ref. 17.) In SSSS and in mouse models of SSSS, ETs diffuse through the entire body, yet have exquisite specificity in causing pathology (e.g., blisters) only in the superficial epidermis. We have hypothesized that this specificity is due to specific recognition and cleavage of a single target, Dsg1.

If ETA binds to Dsg1, and that binding does not require the catalytic serine, then ETA $A_{m u}$ should be a competitive inhibitor of wild-type ETA. Establishing the ability of ETA $A_{m u}$ to protect Dsg1 from cleavage by ETA would also provide an estimate of the dissociation constant $\left(K_{\mathrm{d}}\right)$ for the reaction. To determine the $K_{\mathrm{d}}$ for the interaction between ETA $\mathrm{A}_{\mathrm{mu}}$ and Dsg1, Dsg1 was incubated with increasing concentrations of ETA $A_{m u}$, with ETA $A_{\text {mu }}$ always in large excess of Dsg1. Free Dsg1 was evaluated by relatively rapid treatment with wildtype ETA and by using SDS-PAGE to estimate the fraction cleaved and the fraction protected from cleavage (Figure 3). At concentrations of $13 \mu \mathrm{M}$ and above, ETA
$\mathrm{A}_{\mathrm{mu}}$ mostly inhibited cleavage, but at concentrations of $4 \mu \mathrm{M}$ and below, it did not. Approximately half of the Dsg1 was observed cleaved at an ETA $A_{\text {mu }}$ concentration of $8 \mu \mathrm{M}$, allowing $K_{\mathrm{d}}$ to be roughly estimated as follows:

\section{Equation 1}

$$
\frac{\left[\text { ETA A }_{\text {mu }}\right]_{0}[\mathrm{Dsg} 1]}{\left[\mathrm{ETA} \mathrm{A}_{\mathrm{mu}}-\mathrm{Dsg} 1\right]}=K_{\mathrm{d}}
$$

at $\left[\text { ETA A } A_{\text {mu }}\right]_{0}=8 \mu \mathrm{M},\left[\right.$ Dsg1]/[ETA A $A_{\text {mu }}-$ Dsg1] $\approx 1$,

$$
\text { and } K_{\mathrm{d}} \approx\left[\mathrm{ETA} \mathrm{A} \mathrm{mu}_{0} \approx 8 \mu \mathrm{M}\right.
$$

The magnitude of this $K_{\mathrm{d}}$ is within the range of a hydrolytic reaction whose specificity is due to relatively strong binding in the catalytic site with a relatively low $K_{\mathrm{m}}$, but is probably not consistent with strong binding to an site outside the substrate binding site.

We further showed that mutant ETA binds the epidermis where it causes a blister. This was demonstrated by incubating mouse skin with ETA $A_{m u}$ with a V5 epitope tag on the carboxy terminus at concentrations in excess of $K_{\mathrm{d}}$. Immunofluorescence with antibodies against the V5 tag showed binding of ETA $A_{m u}$ to epidermis in a pattern identical to that of Dsg1 (Figure 4).

Finally, we confirmed by coimmunoprecipitation that ETs bind Dsg1. Incubation of active ETs with Dsg1 showed coprecipitation that quickly decreased with time as cleavage progressed (Figure 5a). The rapid change in the ability to coprecipitate ETA with Dsg1 indicates that ETA dissociates from Dsg1 after hydrolysis of the targeted peptide bond, consistent with

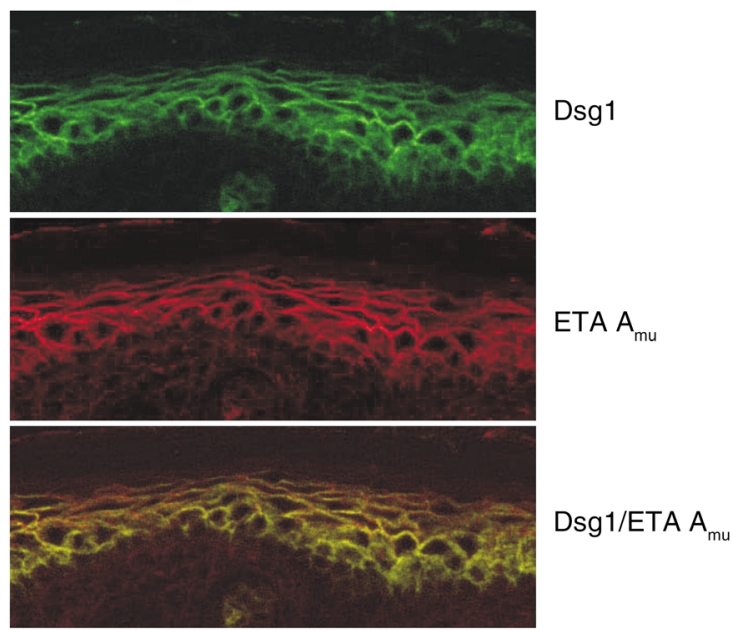

\section{Figure 4}

Immunofluorescence colocalization of ETA $A_{m u}$ and Dsg1 in epidermis. Mouse skin was incubated with ETA $A_{m u}$ with a V5 tag. Double immunofluorescence staining with antibodies against V5 tag (red fluorescence) and Dsg1 (green fluorescence) showed colocalization. Note that immunofluorescence localization of ETA $A_{m u}$ shows the typical pattern of Dsg1 localization, with less intense staining in the basal layer of the epidermis - a pattern opposite that of Dsg3, which is seen most intensely in the basal layer (36). 

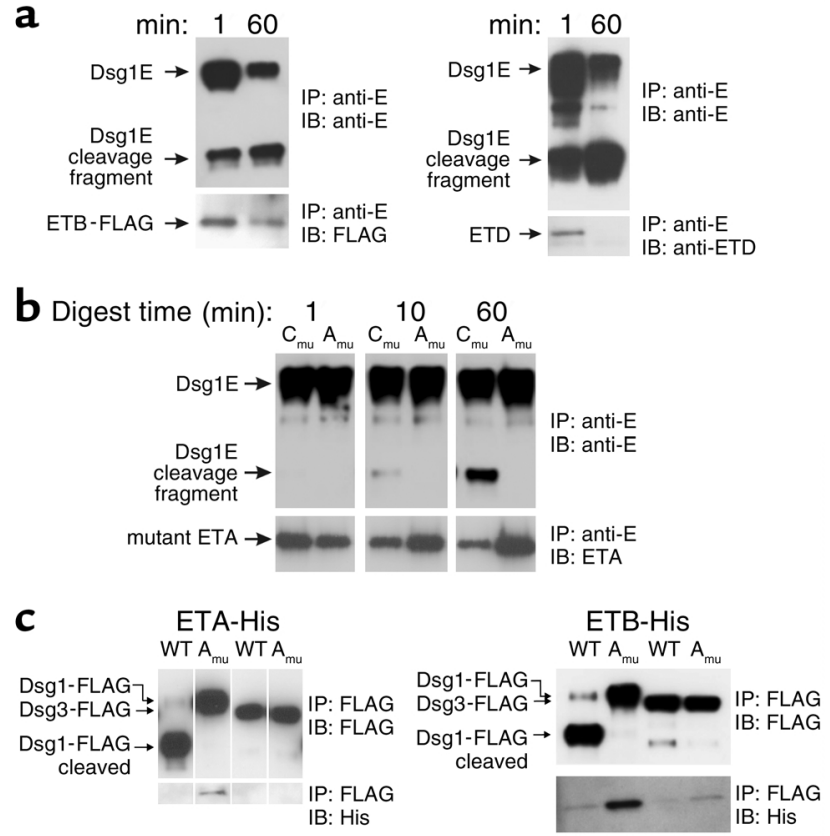

\section{Figure 5}

Coprecipitation of ETs with Dsg1. (a) Immunoprecipitation followed by immunoblotting. Incubation of ETB (marked with a FLAG epitope tag) and ETD with Dsg1 showed coprecipitation at 1 minute that was markedly diminished at 60 minutes, after increased cleavage of Dsg1. Similar results were found for ETA (data not shown). (b) Binding of Dsg1 with ETA $C_{m u}$ and ETA $A_{m u}$. ETA $C_{m u}$, which slowly cleaves Dsg1, showed decreased binding with time. (c) ETA and ETB (marked with His epitope tags) bind to Dsg1 but not Dsg3. These ETs did not cleave or bind to Dsg3, which is highly homologous in amino acid sequence to Dsg1. Similar results were found with ETD (data not shown). IP, immunoprecipitation; IB, immunoblot.

enzyme recycling. Supporting this conclusion are studies with ETA $\mathrm{C}_{\mathrm{mu}}$, which hydrolyzes Dsg1 at a much slower rate. Consistent with the slower cleavage rate, coprecipitation of ETA $\mathrm{C}_{\mathrm{mu}}$ with Dsg1 was observed over a longer time, but still decreased with increasing hydrolysis (Figure $5 \mathrm{~b}$ ). Finally, ETA $\mathrm{A}_{\mathrm{mu}}$, which does not cleave Dsg1 at all, showed increased binding with time.

To show specificity of binding in these studies, we also incubated Dsg3 with ETs and showed that ETs did not cleave or bind to Dsg3, which is highly homologous in amino acid sequence to Dsg1 (Figure 5c).

\section{Discussion}

ETs are serine proteases that specifically bind to and cleave Dsg1 at a unique site after a glutamic acid residue. Previous studies have suggested various possible sites and mechanisms of action of ETs, such as binding to gangliosides, causing release of proteases by keratinocytes; acting as superantigens in stimulating the skin's immune system; and acting as lipases (reviewed in ref. 1). However, recent inferences from crystal structures of ETA and ETB suggest that they might be atypical serine proteases (14-17). These ETs have a structural homology to the chymotrypsin family of serine proteases. The presumptive active site contains the classic catalytic triad of serine 195, histidine 57, and aspartic acid 102 . In addition, homology to the Streptomyces griseus protease Glu-SGP suggests that ETA and ETB cleave after a glutamic acid (or possibly aspartic acid) residue that would be stabilized in the active site by histidine 213 , threonine 190, and lysine 216. However, the ETs are atypical because in ETA, and possibly ETB, the oxyanion hole, which helps to stabilize the transitional-state complex of catalysis, is not properly formed. This is because, as indicated by crystal structures, the peptide bond between proline 192 and glycine 193 is flipped $180^{\circ}$ relative to typical serine proteases. This observation has led to speculation proposing the requirement for interaction with a specific substrate or receptor that would impart the relatively small energy necessary to flip this bond.

Despite these predictions, only one protein substrate, melanocyte-stimulating hormone, was reported to be cleaved by ETs until recently, although an ester substrate was reported $(24,25)$. The biological significance of this protein substrate, however, was not apparent.

Recently we showed that Dsg1 is cleaved by ETA and $\operatorname{ETB}(19,20)$. Here we determine the molecular mechanism of that cleavage and of its exquisite specificity. The ETs (ETA, ETB, and ETD) specifically bind, and consistent with the predictions from amino acid sequence and crystal structure, act as glutamic acid-specific serine proteases to cleave Dsg1 at a unique site. These data are also consistent with previous findings of differential accumulation of ETA in newborn mouse skin compared with blood and other tissues (26).

Proteolysis of this one peptide bond leading to dysfunction of Dsg1 and the desmosome would explain the pathophysiology of blister formation in bullous impetigo and SSSS (see below). It is therefore likely that this peptide bond is critically important to the proper function of Dsg1. Its site in relation to the domain structure of Dsg1 is shown in Figure 6. Interestingly, the ETs cleave at the border between extracellular cadherin domains (ECs) 3 and 4, just toward the carboxy terminal from one of the predicted calcium-binding domains in EC3. Although three-dimensional structures of desmogleins have not been solved, there is marked homology in both calcium-binding sites and ECs between E- and N-cadherins and Dsg1. Structural studies of $\mathrm{E}$ - and $\mathrm{N}$-cadherin have suggested that the border between ECs, through calcium binding, stabilizes the rigidity of the molecule and fixes its orientation, and is critical for function (27-31). The results we present here suggest that this border between ECs in Dsg1 is also critical for their proper function, since the

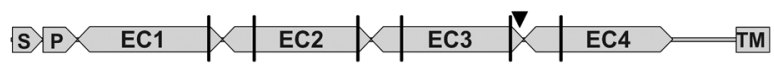

\section{Figure 6}

Schematic diagram of domains and ET cleavage site in Dsg1. Vertical lines indicate presumptive calcium-binding sites. Arrowhead shows ET cleavage site. S, signal peptide; $P$, propeptide sequence; $T M$, transmembrane region. 
cleavage of one peptide bond in this region causes dramatic dysfunction of this desmosomal cadherin.

Pathophysiology of blister localization in PF, bullous impetigo, and SSSS: inactivation of Dsg1 with Dsg3 compensation. In bullous impetigo and SSSS, blisters occur in the superficial epidermis from loss of keratinocyte adhesion in the granular layer. Strikingly, an autoantibodymediated disease, PF, has very similar histopathology to that seen in bullous impetigo and SSSS. PF is caused by anti-Dsg1 autoantibodies (32-34).

Although these antibodies are thought to inactivate Dsg1, which is found throughout the epidermis and in mucous membranes, the blister occurs only in the superficial epidermis. This blister localization has been explained by the "desmoglein compensation hypothesis" (35). This hypothesis states that in areas of epithelium where both Dsg3 and Dsg1 are expressed, a spontaneous blister will not occur when anti-Dsg1 antibodies inactivate Dsg1, because Dsg3 can compensate. However, if only Dsg1 is present, a blister will occur. This hypothesis has been validated both by clinical observation and experimentally. In mucous membranes, both Dsg1 and Dsg3 are found throughout the epithelia; therefore, no blisters are seen in PF, even though anti-Dsg1 antibodies bind to mucous membranes. In epidermis, Dsg1 is found throughout, but Dsg3 is only in the deep epidermis. Therefore, PF antibodies cause only superficial blisters where Dsg1 is not compensated for by Dsg3. Similarly, mothers with PF passively transfer anti-Dsg1 antibodies to their neonates; however, these neonates do not develop PF because neonatal skin, unlike adult skin, expresses Dsg3 throughout all layers (36).

Experimental evidence for the desmoglein compensation hypothesis has been obtained in the neonatal mouse model of PF in which passively transferred antibodies from PF patients cause clinically and histologically typical disease (35). Normal neonatal mice that have Dsg3 in the deep epidermis (similar to adult human epidermis) and throughout the oral mucous membranes develop only superficial epidermal blisters when injected with PF IgG, but Dsg3 knockout mice, which have no Dsg3 to compensate, develop blisters in the deep epidermis and in oral mucous membranes when similarly injected. Furthermore, mice that express Dsg3 in the superficial epidermis (from a transgene containing Dsg3 cDNA driven off an involucrin promoter) are not susceptible to blistering from injected PF IgG (36). These data provide an explanation for antibody inactivation of Dsg1 resulting in blisters only in the superficial epidermis.

ETs cause blisters with pathology identical to those occurring in PF. Although ETs circulate throughout the body in SSSS, as do anti-Dsg1 antibodies in PF, blisters occur only in the superficial epidermis, not in the deep epidermis or in mucous membranes, identical to the findings in PF. Such identical pathology can be explained by the loss of function of Dsg1 caused by antibodies in the case of PF, and by ET cleavage in the case of SSSS or bullous impetigo. Loss of function of
Dsg1 by cleavage with ETs is also consistent with previous immunofluorescence experiments in which ETs cause cellular internalization and loss of cell surface staining of Dsg1 (19).

ETs are skin-smart. A major physiologic function of skin is to provide a barrier against infection. Much of that barrier resides in the stratum corneum. S. aurens, through the use of ETs, has evolved an efficient and extremely focused mechanism of proliferating and spreading under that barrier. Once introduced, the bacteria can spread efficiently by using ET to produce a cleavage plane right under the stratum corneum. To do so, the bacterium has developed a toxin that specifically binds and cleaves a molecule that is critical to adhesion in this area and cleaves it at a specific site that destroys its function.

\section{Acknowledgments}

We thank Michael Plotnik for helpful discussions. This work was supported by grants from NIH and a Grant-inAid for Scientific Research from the Ministry of Education, Science, and Culture of Japan.

1. Ladhani, S., Joannou, C.L., Lochrie, D.P., Evans, R.W., and Poston, S.M. 1999. Clinical, microbial, and biochemical aspects of the exfoliative toxins causing staphylococcal scalded-skin syndrome. Clin. Microbiol. Rev. 12:224-242.

2. Schiavo, G., and van der Goot, F.G. 2001. The bacterial toxin toolkit. Nat. Rev. Mol. Cell Biol. 2:530-537.

3. Darnstadt, G.L. 2000. Cutaneous bacterial infections. In Nelson textbook of pediatrics. R.E. Behrman, R.M. Kliegman, and H.B. Jenson, editors. W.B. Saunders Co. Philadelphia, Pennsylvania, USA. 2028-2030.

4. Shinefield, H.R. 1995. Staphyolococcal infections. In Infectious disease of the fetus and newborn infant. J.S. Remington and J.O. Klein, editors. W.B. Saunders Co. Philadelphia, Pennsylvania, USA. 1105-1141.

5. Cribier, B., Piemont, Y., and Grosshans, E. 1994. Staphylococcal scalded skin syndrome in adults. A clinical review illustrated with a new case. J. Am. Acad. Dermatol. 30:319-324.

6. Gemmell, C.G. 1995. Staphylococcal scalded skin syndrome. J. Med. Microbiol. 43:318-327.

7. Melish, M.E., and Glasgow, L.A. 1970. The staphylococcal scalded-skin syndrome. Development of an experimental model. N. Engl. J. Med. 282:1114-1119.

8. Melish, M.E., and Glasgow, L.A. 1971. Staphylococcal scalded skin syndrome: the expanded clinical syndrome. J. Pediatrics. 78:958-967.

9. Melish, M.E., Glasgow, L.A., and Turner, M.D. 1972. The staphylococcal scalded-skin syndrome: isolation and partial characterization of the exfoliative toxin. J. Infect. Dis. 125:129-140.

10. Lillibridge, C.B., Melish, M.E., and Glasgow, L.A. 1972. Site of action of exfoliative toxin in the staphylococcal scalded-skin syndrome. Pediatrics. 50:728-738.

11. Elias, P.M., Fritsch, P., Dahl, M.V., and Wolff, K. 1975. Staphylococcal toxic epidermal necrolysis: pathogenesis and studies on the subcellular site of action of exfoliatin. J. Invest. Dermatol. 65:501-512.

12. Lee, C.Y., Schmidt, J.J., Johnson-Winegar, A.D., Spero, L., and Iandolo, J.J. 1987. Sequence determination and comparison of the exfoliative toxin A and toxin B genes from Staphylococcus aureus. J. Bacteriol. 169:3904-3909.

13. O'Toole, P.W., and Foster, T.J. 1987. Nucleotide sequence of the epidermolytic toxin A gene of Staphylococcus aureus. J. Bacteriol. 169:3910-3915.

14. Vath, G.M., et al. 1997. The structure of the superantigen exfoliative toxin A suggests a novel regulation as a serine protease. Biochemistry. 36:1559-1566

15. Cavarelli, J., et al. 1997. The structure of Staphylococcus aureus epidermolytic toxin A, an atypic serine protease, at $1.7 \mathrm{~A}$ resolution. Structure. 5:813-824.

16. Vath, G.M., et al. 1999. The crystal structure of exfoliative toxin B: a superantigen with enzymatic activity. Biochemistry. 38:10239-10246.

17. Papageorgiou, A.C., Plano, L.R., Collins, C.M., and Acharya, K.R. 2000. Structural similarities and differences in Staphylococcus aureus exfoliative toxins A and B as revealed by their crystal structures. Protein Sci. 9:610-618.

18. Bailey, C.J., Lockhart, B.P., Redpath, M.B., and Smith, T.P. 1995. The epi- 
dermolytic (exfoliative) toxins of Staphylococcus aureus. Med. Microbiol. Immunol. 184:53-61.

19. Amagai, M., Matsuyoshi, N., Wang, Z.H., Andl, C., and Stanley, J.R. 2000. Toxin in bullous impetigo and staphylococcal scalded-skin syndrome targets desmoglein 1. Nat. Med. 6:1275-1277.

20. Amagai, M., et al. 2002. Staphylococcal exfoliative toxin B specifically cleaves desmoglein 1. J. Invest. Dermatol. 118:845-850.

21. Ishii, K., et al. 1997. Characterization of autoantibodies in pemphigus using antigen-specific enzyme-linked immunosorbent assays with baculovirus-expressed recombinant desmogleins. J. Immunol. 159:2010-2017.

22. Yokosawa, H., Ojima, S., and Ishii, S. 1977. Chemical transformation of the active-site serine residue of Streptomyces griseus trypsin to a cysteine residue. J. Biochem. (Tokyo). 82:869-876.

23. Ladhani, S. 2001. Recent developments in staphylococcal scalded skin syndrome. Clin. Microbiol. Infect. 7:301-307.

24. Rago, J.V., Vath, G.M., Bohach, G.A., Ohlendorf, D.H., and Schlievert, P.M. 2000. Mutational analysis of the superantigen staphylococcal exfoliative toxin A (ETA). J. Immunol. 164:2207-2213.

25. Rago, J.V., et al. 2000. Staphylococcal exfoliative toxins cleave $\alpha$ - and $\beta$ melanocyte-stimulating hormones. Infect. Immun. 68:2366-2368.

26. Fritsch, P., Elias, P., and Varga, J. 1976. The fate of Staphylococcal exfoliatin in newborn and adult mice. Br. J. Dermatol. 95:275-284.

27. Pokutta, S., Herrenknecht, K., Kemler, R., and Engel, J. 1994. Conformational changes of the recombinant extracellular domain of E-cadherin upon calcium binding. Eur. J. Biochem. 223:1019-1026.
28. Nagar, B., Overduin, M., Ikura, M., and Rini, J.M. 1996. Structural basis of calcium-induced E-cadherin rigidification and dimerization. Nature. 380:360-364.

29. Overduin, M., et al. 1995. Solution structure of the epithelial cadherin domain responsible for selective cell adhesion. Science. 267:386-389.

30. Pertz, O., et al. 1999. A new crystal structure, Ca2+ dependence and mutational analysis reveal molecular details of E-cadherin homoassociation. EMBOJ. 18:1738-1747.

31. Tamura, K., Shan, W.S., Hendrickson, W.A., Colman, D.R., and Shapiro, L. 1998. Structure-function analysis of cell adhesion by neural (N-) cadherin. Neuron. 20:1153-1163.

32. Stanley, J.R., Koulu, L., Klaus Kovtun, V., and Steinberg, M.S. 1986. A monoclonal antibody to the desmosomal glycoprotein desmoglein I binds the same polypeptide as human autoantibodies in pemphigus foliaceus. J. Immunol. 136:1227-1230.

33. Koulu, L., Kusumi, A., Steinberg, M.S., Klaus Kovtun, V., and Stanley, J.R. 1984. Human autoantibodies against a desmosomal core protein in pemphigus foliaceus. J. Exp. Med. 160:1509-1518.

34. Amagai, M., Hashimoto, T., Green, K.J., Shimizu, N., and Nishikawa, T. 1995. Antigen-specific immunoabsorption of pathogenic autoantibodies in pemphigus foliaceus. J. Invest. Dermatol. 104:895-901.

35. Mahoney, M.G., et al. 1999. Explanations for the clinical and microscopic localization of lesions in pemphigus foliaceus and vulgaris. J. Clin. Invest. 103:461-468.

36. Wu, H., et al. 2000. Protection of neonates against pemphigus foliaceus by desmoglein 3. N. Engl.J. Med. 343:31-35. 